\title{
Avatare: Parasoziale Beziehungen zu virtuellen Akteuren
}

\author{
Tilo Hartmann / Christoph Klimmt / Peter Vorderer
}

Mit der Verbreitung interaktiver Medienangebote sind auch virtuelle Charaktere, so genannte Avatare, entstanden. Avatare arbeiten zum Beispiel als automatische Assistenten auf Internetseiten. Andere werden zu virtuellen "Stars" aufgebaut, die in verschiedenen Medien erscheinen. Der Beitrag stellt das Phänomen "Avatare "vor und diskutiert die Frage, wie sie von Mediennutzer/innen wabrgenommen werden. Dabei wird auf das Konzept der parasozialen Beziehungen von Horton E Wobl zurückgegriffen. Anschließend werden zwei voneinander unabhängig durchgefübrte Befragungsstudien vorgestellt, welche die Art und das Ausmaß der parasozialen Bindungen zwischen Mediennutzer/innen und verschiedenen Avataren beleuchten. Vor dem Hintergrund dieser Ergebnisse und der prognostizierten Evolution virtueller Akteure werden abschließend Desiderata für die künftige medien- und kommunikationswissenschaftliche Forschung diskutiert.

\section{Avatare - die Bewohner der digitalen Welt}

Wer heute im Internet surft, Virtual-Reality-Umgebungen aufsucht oder Computerspiele spielt, trifft immer häufiger auf künstliche Personen, die sich als "Assistenten“, „Freunde“ oder „Stellvertreter“ anbieten. Die Rede ist von „Avataren“. Ursprünglich stammt der Begriff „Avatar“ aus dem Sanskrit und bezeichnet eine Gottheit, die sich vorübergehend zu den Menschen herabgelassen hat (Klussmann, 2000). Im Kontext der Neuen Medien wurde er durch den Science-Fiction-Roman „Snowcrash“ von Neal Stephenson (1995) populär, in dem Begegnungen in virtuellen Räumen mit Hilfe von Avataren, „Stellvertretern“ im Netz, stattfinden. So stand der Begriff „Avatar“ zunächst ausschließlich für grafische Repräsentationen von Nutzern in Chat-Rooms (Trzka, 1998), Multi-User-Dungeons (MUDs; vgl. Krotz, 1996), Online-Spielen (Schmidt, 1998) und Virtual-Reality-Umgebungen (Ueberhorst, 1995). Entsprechend definiert Döring (1999, S. 98) Avatare als „Stellvertreter, virtuelle Repräsentanten der materiegebundenen Wesen, die sie ins Leben gerufen haben“.

Im Zuge der technischen Weiterentwicklung interaktiver Medien, insbesondere von Computerspielen und dem World Wide Web, entstanden in den vergangenen Jahren jedoch auch neue Formen von Avataren (vgl. Abbildung 1). Ihre Evolution vollzog sich weg von der „elektronischen Marionette“ (Fritz, 2000, S. 12), die vollkommen von der Steuerung durch wirkliche Menschen abhängig ist, hin zum „virtual actor“ (Lombard \& Ditton, 1997, o. S.), der dank „künstlicher Intelligenz“ selbstständig mit Mediennutzern „interagieren“ kann. Damit einher geht die Entwicklung und Veränderung der äußeren Erscheinung von Avataren. Mit aufwendiger Technik wird das Aussehen der künstlichen Charaktere mittlerweile so „realistisch“ und attraktiv wie möglich gestaltet (vgl. Bente \& Otto, 1996).

Oftmals dienen „intelligente“ Avatare als Helfer, etwa indem sie den Besuchern einer Webseite bei der Navigation assistieren oder den Kunden einer Internet-Bank als „Berater" zur Seite stehen. Neben diesen instrumentellen Einsatzformen existieren aber auch Versuche, Avatare als „Stars“ oder „Marken“ aufzubauen, die in verschiedenen Medienangeboten und Themenumfeldern präsent sind und die innerhalb bestimmter 
Abbildung 1: Populäre Avatare. Von links nach rechts: E-Cyas (von www.e-cyas.com), Kyoko Date (von www.horipro.co.jp) und Lara Croft (von www.tombraider.com)
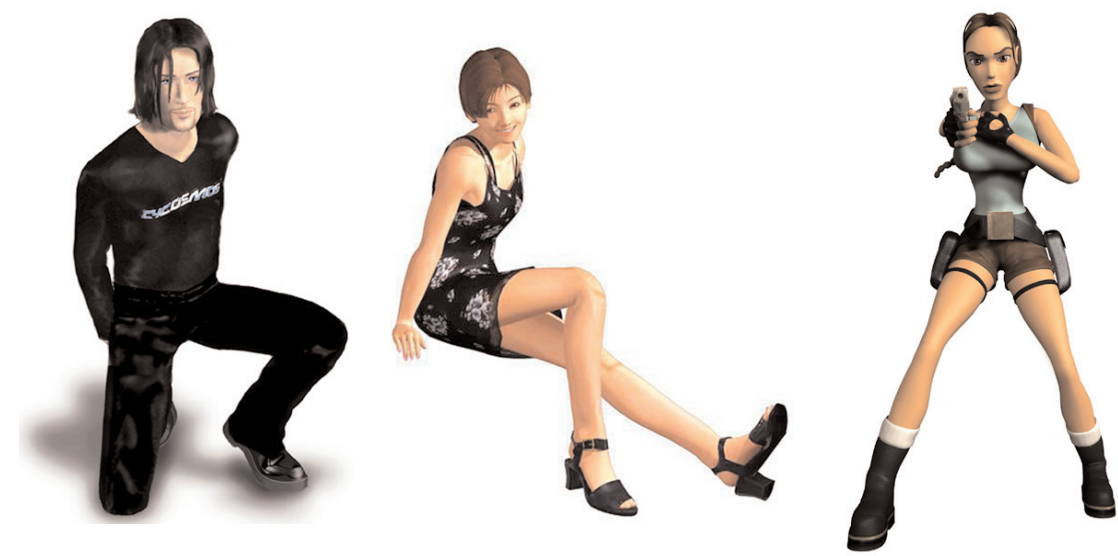

Zielgruppen große Popularität erreichen sollen. Beispiele sind die Computerspiel-Figur „Lara Croft" und der Internet-Avatar „E-Cyas“ (Endo-Cybernetic Artificial Star, vgl. Schmidt, 1998). Sie treten beide nicht nur in ihrer jeweiligen digitalen „Heimat“ auf, sondern erscheinen auch in Medienumfeldern, die üblicherweise Prominenten aus Fleisch und Blut vorbehalten sind, so zum Beispiel in Musikvideos, Fernsehshows oder Publikumszeitschriften. Fiktive Lebensläufe und Persönlichkeitsprofile sollen den virtuellen Stars „Charakter" und „Tiefe“ verleihen. Bekannte und beliebte Avatare lassen sich von ihren Eigentümern für unterschiedlichste Zwecke einsetzen. Zum Aufgabenspektrum von Star-Avataren gehören unter Anderem Werbespots, die „Arbeit“ als Fotomodell und Auftritte als Moderator(in) in unterschiedlichen Medienangeboten (Jöckel, 1997; Höflich, 1998; Blittkowsky, 1999).

Über diese Entwicklungen darf allerdings nicht übersehen werden, dass sich die bislang existierenden Avatare sehr deutlich von menschlichen Prominenten unterscheiden. Die Fähigkeit, mit Mediennutzern sozial zu interagieren, ist in den meisten Fällen noch nicht sehr weit entwickelt und muss oftmals dadurch kompensiert werden, dass die angeblichen Kommunikate des Avatars in Wahrheit von Redakteuren verfasst werden. Von vollkommen selbstständigen virtuellen Figuren kann also noch keine Rede sein, wenngleich rudimentäre Formen der Interaktion bereits ohne menschliche Steuerung des Avatars möglich sind.

Vor dem Hintergrund der Existenz und Evolution virtueller Akteure stellt sich die Frage, wie die Nutzer interaktiver Medienangebote mit Avataren umgehen. Werden Avatare als echte Stars betrachtet, die man bewundern und bejubeln kann? Oder gelten sie als virtuelle Abziehbilder, die zu „dumm“ und „unecht“ wirken, als dass man sie ernst nehmen könnte? Diese Fragen werden im Folgenden erörtert. Als theoretischer Rahmen bietet sich das ursprünglich kommunikationswissenschaftliche Konzept der parasozialen Interaktionen und Beziehungen an, das die Relation zwischen Mediennutzern und Medienfiguren beschreibt und erklärt (vgl. unten: 2.). Nach einer kurzen Einführung in dieses Konzept werden zwei unabhängig voneinander entstandene empirische Studien über parasoziale Beziehungen zu Avataren vorgestellt, die erste Hinweise 
zu der Frage liefern, inwiefern Mediennutzer Avatare schon heute als eigenständige soziale Persönlichkeiten auffassen und sie als Interaktionspartner ernst nehmen (vgl. unten: 3.). Zum Abschluss werden Perspektiven aufgezeigt, wie die Erforschung virtueller Akteure und ihrer Bedeutung für die Rezipienten theoretisch und methodisch weitergeführt werden könnte (vgl. unten: 4.).

\section{Parasoziale Interaktionen und Beziehungen}

\subsection{Das interaktionistische Rezeptionsverständnis von Horton und Wobl und seine Wei- terentwicklungen}

Das auf Horton und Wohl (1956; Horton \& Strauss, 1957) zurückgehende Konzept der parasozialen Interaktionen und Beziehungen beschreibt das Phänomen, dass Rezipienten mit einer medial (durch Radio, Fernsehen und Film) vermittelten Figur, genannt „Persona“, sozial interagieren. Als Persona betrachteten Horton und Wohl hauptsächlich Menschen, die in nicht-fiktionalen Fernsehsendungen agieren, wie Quiz-Master, Ansager und Interviewer. Ausgangspunkt der sozialen Interaktion zwischen Persona und Rezipienten ist die „illusion of a face-to-face relationship“ (Horton \& Wohl, 1956/1986, S. 185). Obwohl die „Interaktionspartner" durch die mediale Vermittlung voneinander getrennt sind, verhalten sie sich - so die Annahme - wie in einer sozialen Kommunikationssituation:

Die Medienfiguren sprechen die Rezipienten an, als ob sie sie sehen könnten, verwenden mimische und gestische Signale der interpersonalen Kommunikation, laden die Zuschauer zur Teilnahme an der Interaktion ein und versuchen, den Eindruck eines positiven, geradezu freundschaftlichen Verhältnisses zwischen sich selbst und dem Publikum zu vermitteln. Neben solchen direkt auf das (vermutete) Publikum bezogenen Interaktionsangeboten trägt auch die Kommunikation der Akteure innerhalb des Medienangebots zur Überwindung der Wahrnehmung medialer Vermittlung und damit zur Herstellung einer scheinbar sozialen Relation zwischen Persona und Rezipienten bei: „The most usual way of achieving this ambiguity is for the persona to treat his supporting cast as a group of close intimates. ... The member of the audience, therefore, ... tends to believe that this fellowship includes him by extension" (Horton \& Wohl, 1956/1986, S. 189). Darüber hinaus wird versucht, über Darstellungseffekte, wie zum Beispiel durch Nahaufnahmen der Personae, die Illusion von Nähe zum Publikum zu erzeugen (vgl. auch Meyrowitz, 1978).

Die Rezipienten sind also ein eingeplanter Teil der medialen Inszenierung. Ihnen wird von den Personae des Medienangebots eine Rolle innerhalb des Geschehens nahe gelegt. Ob sie diese „appropriate answering role“ (Horton \& Wohl, 1956/1986, S. 191) akzeptieren, können die Zuschauer oder Hörer jedoch selbst entscheiden. Lassen sie sich auf das Angebot ein, erleben sie die Rezeption wie eine soziale Interaktion mit den Medienfiguren: Sie ist geprägt durch „Prozesse der Personenwahrnehmung und -beurteilung, Attributionen, soziale Vergleiche und Validierungen, Verhaltensantizipationen und personenbezogene innere Verbalisierungen" (Gleich, 1997, S. 41).

In dieser Hinsicht ist der Umgang der Rezipienten mit Medienfiguren eine Spielart der normalen zwischenmenschlichen Interaktion (Horton \& Wohl, 1956; Gleich, 1996). Para-sozial ist diese Form der Interaktion, weil die „Anwesenheit“ der Personae nur medial vermittelt ist, die Personae also die Gegenwart und Aufmerksamkeit von Interaktionsteilnehmern nur antizipieren können, und weil es - zumindest bei den nicht-interaktiven Medien - keinen Rückkanal von den Zuschauern zu den Medienfiguren gibt. 
Insofern erscheint es notwendig, „zwischen unmittelbarer, zweiseitiger Face-to-FaceKommunikation und mittelbarer, einseitiger Kommunikation mit Medienfiguren" (Keppler, 1996, S. 11, Hervorhebung im Original) zu trennen. Denn zum einen gehen mit der medialen Vermittlung der sozialen Situation bestimmte Beschränkungen und Eigenheiten der Interaktion einher, zum anderen spielt das Wissen um die Medialität der Situation für das Erleben der Zuschauer eine entscheidende Rolle (Wulff, 1996). So ist es den Zuschauern zum Beispiel möglich, die ihnen zugedachte Rolle zurückzuweisen. Dadurch eröffnen sich neue Wege, die soziale Situation zu „lesen“, zum Beispiel durch eine kritische Abschätzung der Leistung einer Persona oder durch eine hämisch-herablassende Sichtweise „dilletantischer“ Auftritte von „Möchtegern-Stars“ (vgl. auch Vorderer \& Knobloch, 1996). In echten Kommunikationssituationen sind solche Verhaltensweisen sanktioniert. Weil die Zuschauer aber wissen, dass die Personae ihre Reaktionen nicht registrieren, können sie bei parasozialen Interaktionen im Vergleich zu ortho-sozialen Interaktionen zusätzliche Freiheitsgrade ausnutzen und genießen. Aber auch wenn die Rezipienten die angebotene Rolle innerhalb der parasozialen Interaktion akzeptieren und selbst wenn ihnen die Medialität der Situation bewusst bleibt (Hippel, 1993; Wulff, 1996), können die Zuschauer das „conversational give and take“ (Horton \& Wohl, 1956/1986, S. 186) einer parasozialen Interaktion wie eine wirkliche soziale Situation erleben.

Aus einer Reihe parasozialer Interaktionen zwischen einem Rezipienten und einer Persona entwickelt sich in der Wahrnehmung des Rezipienten eine andauernde Beziebung zur Persona. Entsprechend wird „die unmittelbare, während der Rezeption stattfindende ,Begegnung' zwischen Rezipient und Medienakteur als parasoziale Interaktion bezeichnet und die über die einzelne ,Begegnung' hinausgehende Bindung des Zuschauers an eine Persona als parasoziale Beziebung" (Vorderer, 1998, S. 698, Hervorhebung im Original). Sowohl bei Horton und Wohl (1956) als auch im überwiegenden Teil der an ihnen orientierten kommunikationswissenschaftlichen Forschung wurden die Konstrukte „parasoziale Interaktion“ und „parasoziale Beziehungen“ nicht ausreichend präzise voneinander getrennt. In vielen Studien wurden die beiden Begriffe synonym verwendet (z. B. Rubin \& McHugh, 1987; Perse \& Rubin, 1989). Auch das am häufigsten verwendete Instrument zur Messung parasozialer Bindungen von Rubin, Perse \& Powell (1985) heißt „Parasocial Interaction Scale“, misst aber zumindest auch in Teilen parasoziale Beziehungen (Gleich, 1997). Hippel (1992) und Gleich (1996; 1997) argumentieren jedoch wie Vorderer (1998), dass es durchaus sinnvoll, wenn nicht gar notwendig ist, die beiden Begriffe inhaltlich voneinander zu unterscheiden. Denn die über die einzelnen Rezeptionssituationen hinweg existierenden parasozialen Beziehungen gelten als ein zentrales Motiv für die regelmäßige Zuwendung zu bestimmten Medienangeboten wie Nachrichtensendungen (Rubin, Perse \& Powell, 1985), TV-Serien (Vorderer, 1996b) oder Talkshows (Trepte, Zapfe \& Sudhoff, im Druck): Sie können das Beziehungsnetzwerk von Mediennutzern bereichern und darüber hinaus für schüchterne (Vorderer \& Knobloch, 1996) oder einsame (Fabian, 1993) Rezipienten sogar als Ersatz für echte Sozialkontakte dienen.

\subsection{Parasoziale Beziehungen zu virtuellen Akteuren}

Wenn die parasozialen Bindungen an Figuren aus „klassischen“ Medienangeboten eine so zentrale Rolle für die Zuwendung der Rezipienten zu diesen Angeboten spielen, liegt es nahe, das Konzept auch auf virtuelle Akteure in den „neuen“ Medien anzuwenden. Denn die Schöpfer von Avataren versuchen, sie als getreue Abbilder echter Menschen 
zu gestalten, beispielsweise durch fotorealistische Gesichtszüge, „natürliche“ Gesten, die direkte Ansprache der Nutzer oder durch E-Mail- und Chat-Kommunikation (z. B. Snoddy, 2000). Die Zielsetzung, Star-Avataren eine möglichst große Attraktivität für parasoziales Beziehungshandeln der Rezipienten zu verleihen, ist deutlich erkennbar. Entsprechend lässt sich die eingangs formulierte Fragestellung präzisieren: Wie stellen sich parasoziale Beziehungen zu den heute existierenden Avataren dar? Welche Personen entwickeln besonders starke parasoziale Bindungen an Avatare? Gibt es Unterschiede zwischen Avataren und Fernsehfiguren bezüglich der Bindungsintensität und -qualität?

Obwohl bereits Horton und Wohl (1956/1986, S. 186) parasoziale Bindungen nicht nur für menschliche Personae, sondern auch für „puppets ... anthropomorphically transformed into ,personalities“" angenommen haben, ist es offensichtlich, dass Avatare, sollen sie als "Anbieter“ parasozialer Beziehungen fungieren, wesentlich stärkere Barrieren in der Wahrnehmung und den Einstellungen der Rezipienten überwinden müssen als Personen aus dem Fernsehen. So könnte die Virtualität der Avatare ein erstes Hindernis für die Entstehung parasozialer Bindungen sein. Denn damit solche Bindungen zustande kommen, müssen die virtuellen Akteure zunächst einmal von den Rezipienten als „soziale Wesen“ eingestuft werden. Was aber spricht dafür, dass die noch immer deutlich als Computergraphiken erkennbaren Avatare im Auge des Betrachters als „Personen“ und nicht als „Dinge“ erscheinen? Reeves und Nass (1996) konnten zeigen, dass schon schwache Anzeichen sozialen Verhaltens seitens eines Computers genügen, um seine Nutzer zu veranlassen, mit ihm ähnlich wie mit einem Menschen zu interagieren (vgl. auch Moon \& Nass, 1996; Lombard \& Ditton, 1997). Zu ähnlichen Ergebnissen kommen die ersten Erprobungen von künstlichen „Agenten“ in Virtual-Reality-Umgebungen (z. B. Bates, 1994; Reilly, 1996; Robinson 1997).

Ein zweites Problem, das Avatare überwinden müssen, damit parasoziale Bindungen $\mathrm{zu}$ den Mediennutzern entstehen, ist ihre eingeschränkte Kommunikationsfähigkeit. Nur wenn eine vergleichsweise „natürliche“ Form der Konversation mit einem virtuellen Charakter möglich ist, hat er die Chance, als Gegenstand von Beziehungshandlungen ausgewählt zu werden. Schon seit den 50er Jahren des vergangenen Jahrhunderts wird an der kommunikativen Kompetenz von Computerprogrammen gearbeitet (Turing, 1950), wobei mittlerweile auch paraverbale Kommunikationsformen simuliert werden (Bente \& Otto, 1996; Vilhjálmsson \& Cassel, 1998). Die Erfolge dieser Bemühungen dokumentieren Evaluationsstudien wie die von Cassel und Vilhjálmsson (1999), in der Versuchspersonen einen Avatar mit weiter entwickelten Kommunikationsfähigkeiten „natürlicher“ fanden als einen virtuellen Akteur, der mit weniger Konversationskompetenz ausgestattet war. Insofern scheinen die technischen Voraussetzungen für eine parasoziale Beziehung zwischen Mediennutzern und virtuellen Akteuren auf Seiten der Avatare gegeben zu sein (vgl. auch Bente, Petersen, Krämer \& Buschmann, 1999).

Entsprechend hat es bereits einige Versuche gegeben, das Verhalten von Mediennutzern gegenüber virtuellen Akteuren als parasoziales Beziehungshandeln zu konzeptualisieren und empirisch abzubilden. Krotz (1996, S. 89) weist darauf hin, dass sich durch Virtual-Reality-Technologien neue „Kommunikationsformen“ entwickeln, die als „Ausdifferenzierung zwischen sozialer und parasozialer Interaktion“ (ebd.) verstanden werden können, und dass der Zugang Horton und Wohls geeignet sei, sich der Interaktion mit den neuen Medien und ihren Personae zu nähern. Bente und Otto (1996) skizzieren die Möglichkeiten, nonverbales Kommunikationsverhalten mit virtuellen Figuren $\mathrm{zu}$ simulieren und parasoziale Interaktionen zwischen VR-Nutzern und diesen 
Figuren anzustoßen. Mit einer empirischen Untersuchung weisen sie nach, dass die Mediennutzer das Verhalten der virtuellen Akteure ähnlich wie das echter Interaktionspartner bewerten. Und Rettberg (1999) befasst sich mit dem „Star-Kult“ um Lara Croft und führt die Popularität der Videospiel-Heldin auf die parasozialen Bindungen der Spieler zurück.

\section{Zwei empirische Studien zu parasozialen Beziehungen mit virtuellen Akteuren}

Trotz der oben erwähnten theoretischen und empirischen Ansätze, das Konzept der parasozialen Beziehungen auf virtuelle Akteure anzuwenden, steht die Forschung zur sozialen Bedeutung von Avataren noch am Anfang. Im Folgenden werden zwei Befragungsstudien vorgestellt, welche die parasozialen Bindungen an Avatare wie E-Cyas und Lara Croft beleuchten.

\section{Studie 1}

\section{Fragestellung}

Im Mittelpunkt der Untersuchung standen so genannte Star-Avatare, also virtuelle Akteure, die nicht als Assistenten oder Helfer, sondern als eigenständige Persönlichkeiten konzipiert sind. Star-Avatare werden von ihren Schöpfern hauptsächlich für Maßnahmen im Bereich der Produktvermarktung und Kundenbindung eingesetzt (vgl. oben: 1.). Es stellt sich daher die Frage, ob sich eine empirische Anwendung des Konzepts der parasozialen Beziehungen auf virtuelle Prominente als fruchtbar erweist und wie sich die parasozialen Beziehungen zu Star-Avataren darstellen. Denn es ist nach wie vor unklar, ob „VR-Bewohner ebenso zu unserem Bekanntenkreis zählen [werden] wie wirkliche Personen“ (Bente \& Otto, 1996, S. 219). Diese bedeutende Rolle im Beziehungsnetzwerk von Mediennutzern wird zumindest Fernsehcharakteren zugeschrieben (Gleich, 1996; 1997) und wird zweifelsohne auch von den Unternehmen, die hinter den StarAvataren stehen, angestrebt (Jöckel, 1997). Außerdem sollte untersucht werden, inwiefern sich weibliche und männliche Internetnutzer hinsichtlich der Beziehungsintensität $\mathrm{zu}$ „männlichen“ und „weiblichen“ Avataren unterscheiden. Interaktionseffekte zwischen dem Geschlecht der Rezipienten und dem Geschlecht der Persona haben sich bereits in früheren Studien als relevant für das Ausmaß der parasozialen Bindungen erwiesen (Vorderer \& Knobloch, 1996). Daher sollte geprüft werden, ob sich dieses Muster auch in den Beziehungen zu Avataren wiederfinden lässt.

\section{Methode}

In einer explorativ angelegten Online-Befragung wurde untersucht, wie viele Star-Avatare den Teilnehmern bekannt sind, und wie intensiv ihre parasoziale Bindung zu ihrem bevorzugten Avatar ausfällt. Die Kenntnis von Star-Avataren wurde mittels einer Liste von 15 virtuellen Akteuren abgefragt; ein nicht existierender Avatar („Leila Loo“) wurde aufgeführt, um die Sorgfalt der Teilnehmer bei der Kenntnisabfrage zu kontrollieren. Außerdem bestand die Möglichkeit, bis zu drei weitere Avatare selbstständig zu ergänzen. Weiterhin wurde erhoben, wie attraktiv der Avatar in den Augen der Teilnehmer ist. Diese Frage ist nach den Erkenntnissen der bisherigen Forschung relevant für das Ausmaß und die Qualität von parasozialen Beziehungen (Gleich, 1997). Sie wurde mit dem fünffach gestuften Item „Ich finde, [Name] ist attraktiv“ operationalisiert, wobei 
die Endpunkte mit „stimme nicht zu“ bzw. „stimme voll und ganz zu“ bezeichnet waren. Zur Messung der parasozialen Beziehungen wurde eine modifizierte Variante der Parasocial-Interaction-Scale (Rubin, Perse \& Powell, 1985) eingesetzt. Sie ist ein häufig verwendetes, wenn auch wiederholt kritisiertes (Gleich, 1997) Instrument zur Erfassung von parasozialen Interaktionen und Beziehungen. Einer der Hauptkritikpunkte ist genau diese fehlende Differenzierung zwischen Interaktion und Beziehung. Dennoch wurde die Skala als Grundlage für die Messung parasozialer Bindung herangezogen, allerdings um einige Items vekürzt und in Teilen umformuliert, damit die Items besser auf den Kontext der Star-Avatare anwendbar waren (vgl. ausführlich zur Skalenbildung: Hartmann, 2001). Die Endpunkte der Likert-Skala waren mit „trifft überhaupt nicht zu“ bzw. „trifft voll und ganz zu“ benannt. Auf die Befragung wurde in Internetangeboten, die eine thematische Nähe zu Avataren besitzen, hingewiesen, zum Beispiel auf www.cycosmos.de, der „Heimat“ von E-Cyas. Über einen als „Umfrage über Avatare“ betitelten Hyperlink konnten interessierte Personen zu dem Online-Fragebogen gelangen. Die Stichprobe war also selbst rekrutiert.

\section{Ergebnisse}

Insgesamt nahmen 422 Personen an der Befragung teil. 58 Prozent der befragten Personen waren weiblich, 41 Prozent männlich. Das Durchschnittsalter betrug etwa 23 Jahre $(M=22.80, S D=7.29)$. Vergleicht man die Stichprobe hinsichtlich dieser Merkmale mit der Gesamtheit der deutschen Internet-Nutzer, wie sie in der ARD/ZDF-Online-Studie (van Eimeren \& Gerhard, 2000) beschrieben wird, so entspricht die Altersverteilung in der Stichprobe ungefähr der Verteilung in der Online-Nutzerschaft; jedoch sind Frauen in der Stichprobe im Vergleich zu den Ergebnissen der ARD/ZDF-Studie deutlich überrepräsentiert.

Kenntnis und Beliebtheit von Avataren. 19 Personen gaben an, den nicht existierenden Kontroll-Avatar zu kennen. Deshalb wurden auch ihre sonstigen Angaben zur Kenntnis von Avataren nicht berücksichtigt. Insgesamt sind den Befragten durchschnittlich zwei Avatare $(M=2.06, S D=1.35)$ bekannt. Am häufigsten wurden die StarAvatare Lara Croft (72 Prozent Bekanntheitsgrad), E-Cyas (68 Prozent) und E-ve (21 Prozent) genannt. Als Lieblings-Avatare wurden hauptsächlich Lara Croft (43 Prozent der Stichprobe) und E-Cyas (36 Prozent) ausgewählt; die restlichen Nennungen bezogen sich entweder auf weniger bekannte Star-Avatare oder aber auf Helfer-Avatare (vgl. oben: 1.), die im vorliegenden Kontext nicht relevant sind. E-Cyas scheint insbesondere bei weiblichen Internetnutzern beliebt zu sein: 76 Prozent der Teilnehmer, die ECyas als Lieblings-Avatar nannten, waren weiblich (bei Lara Croft: 47 Prozent). Drei Viertel der Befragten hatten ihren Lieblings-Avatar im vergangenen halben Jahr in Zeitschriften angetroffen; 63 Prozent waren ihm bzw. ihr im Internet begegnet, und an dritter Stelle folgt das Fernsehen (49 Prozent). Am häufigsten begegneten die Befragten ihrem Lieblings-Avatar in „sonstigen“ Medienangeboten (durchschnittlich 16.25 Mal $[S D=23.80]$ in den vergangenen sechs Monaten), in "Software“-Programmen (14.54 $\mathrm{Mal}[\mathrm{SD}=23.20])$ und im Internet (13.04 Mal [SD = 19.60]).

Parasoziale Beziebungen zu Avataren. Über alle genannten Lieblings-Avatare hinweg erreicht die Skala zur parasozialen Beziehung einen vergleichsweise hohen internen Reliabilitätswert (Cronbachs $\alpha=.94$ ), ist somit also gut einsetzbar. Fasst man die Skala zu einem Mittelwert-Index zusammen, ergibt sich eine sehr schwache durchschnittliche Beziehungsintensität. Der Mittelwert liegt deutlich unter dem Skalenmittelpunkt $(M=$ $1.77, S D=.82$; Skalenmittelpunkt $=3$ ). Die beiden am häufigsten ausgewählten Avatare 
Lara Croft $(M=1.86, S D=.81)$ und E-Cyas $(M=1.77 ; S D=.85)$ unterscheiden sich hinsichtlich der mittleren Beziehungsintensitäten sowohl vom Gesamtmittelwert als auch untereinander $(t(295)=.56, n s)$ nur in geringem Maße.

Dimensionen derparasozialen Beziebung zu Avataren. In verschiedenen Studien über parasoziale Beziehungen (z. B. Vorderer, 1996a; Visscher \& Vorderer, 1998; Gleich, 1997) hat sich die Skala zur Messung parasozialer Bindungen als mehrdimensional erwiesen. Deshalb wurde auch in der vorliegenden Studie eine Faktorenanalyse (Hauptkomponentenanalyse mit VARIMAX-Rotation) durchgeführt, um die inhaltliche Qualität der parasozialen Bindung an Avatare differenzierter erfassen zu können (vgl. Tabelle 1). Die Faktorenanalyse parasozialer Beziehungen ergab zwei Hauptkomponenten, die zusammen 63 Prozent der Varianz aufklären. Fünf Items, die keinem der beiden Faktoren trennscharf zugeordnet werden konnten, wurden ausgeschlossen. Der erste Faktor umschreibt eine enge freundschaftliche Beziehung, in der die Medialität des Lieblings-Avatars von den Nutzern weitestgehend ausgeblendet wird. Personen, die hohe Werte auf dieser Dimension erreichen, betrachten also den Avatar als „echten“

Tabelle 1: Mittlere Zustimmung (auf einer Skala von 1-5), Standardabweichungen und Faktorenladungen der Items auf den zwei Dimensionen parasozialer Beziebung. Es werden nur Faktorladungen $>.45$ dargestellt.

\begin{tabular}{|c|c|c|c|c|}
\hline \multirow[t]{2}{*}{ Item } & \multirow{2}{*}{\multicolumn{2}{|c|}{$\begin{array}{l}\text { M } \\
(\mathrm{n}=320)\end{array}$}} & \multicolumn{2}{|c|}{ Faktorladungen } \\
\hline & & & Faktor & Eal tor \\
\hline $\begin{array}{l}\text { Manchmal passiert es mir, dass ich in Gedanken oder auch } \\
\text { tatsächlich irgendetwas zu [Name] sage }\end{array}$ & 1.29 & 0.84 & .83 & \\
\hline Ich glaube, [Name] und ich sind uns ziemlich ähnlich & 1.41 & 0.91 & .82 & \\
\hline $\begin{array}{l}\text { Wenn ich [Name] sehe, kommt es mir vor, als wenn ich mit } \\
\text { Freunden zusammen wäre, dann fühle ich mich wohl }\end{array}$ & 1.50 & 1.03 & .77 & \\
\hline $\begin{array}{l}\text { Auch wenn ich gerade nicht auf der Homepage von [Name] } \\
\text { bin, versuche ich, möglichst viel über [Name] zu erfahren, } \\
\text { um sie/ihn noch besser kennen zu lernen }\end{array}$ & 1.42 & 0.90 & .74 & \\
\hline $\begin{array}{l}\text { Wenn ich [Name] begegne, kann ich mir immer gut ein Bild } \\
\text { über sie/ihn machen, z. B. über ihre/seine Persönlichkeit }\end{array}$ & 1.67 & 1.10 & .69 & \\
\hline In manchen Situationen fühle ich mich an [Name] erinnert & 1.85 & 1.21 & .59 & \\
\hline $\begin{array}{l}\text { Wenn [Name] auf einem bestimmten Internetangebot } \\
\text { auftauchen würde, dann würde ich mir dieses Internet- } \\
\text { angebot bestimmt anschauen }\end{array}$ & 2.03 & 1.24 & & .82 \\
\hline $\begin{array}{l}\text { Ich finde es angenehm, das Bild von [Name] zu Hause } \\
\text { auf meinem Bildschirm zu sehen }\end{array}$ & 2.17 & 1.29 & & .81 \\
\hline $\begin{array}{l}\text { Wenn in Zeitungen oder Zeitschriften etwas über [Name] } \\
\text { stünde, würde ich es auf jeden Fall lesen }\end{array}$ & 2.33 & 1.37 & & .73 \\
\hline $\begin{array}{l}\text { Wenn [Name] seine/ihre Meinung oder auch persönlichen } \\
\text { Gefühle zum Ausdruck bringt, ist das jeweilige (Internet-) } \\
\text { Angebot für mich attraktiver }\end{array}$ & 1.81 & 1.19 & & .70 \\
\hline $\begin{array}{l}\text { Mir würde sofort auffallen, wenn [Name] mal nicht wie } \\
\text { gewohnt auf einem Internetangebot auftauchen würde }\end{array}$ & 2.12 & 1.27 & & .70 \\
\hline $\begin{array}{l}\text { Wenn es möglich wäre, würde ich [Name] gerne einmal } \\
\text { persönlich kennen lernen }\end{array}$ & 1.98 & 1.39 & & .53 \\
\hline
\end{tabular}


Freund, dessen Virtualität einer engen Verbundenheit nicht im Wege steht. Der Faktor wird entsprechend als quasi-reale Beziehungsdimension bezeichnet; er erklärt 32 Prozent der Gesamtvarianz. Demgegenüber entspricht der zweite Faktor einer medialen Beziehungsdimension, denn er umfasst Items, welche die Vermitteltheit und Künstlichkeit des Avatars in den Vordergrund stellen. Diese Medialität ist ein bewusst erlebter Bestandteil der Relation zum virtuellen Akteur. Für Personen, die hohe Werte auf der zweiten Dimension erreichen, besitzen demzufolge Avatare eher ästhetische als soziale Qualität. Die Beziehung zum Avatar ist durch das Wissen um die Irrealität des Avatars geprägt: Er kann zwar interessant sein und zu wiederholter Interaktion einladen, doch er bleibt an sein Medium gebunden. Der Faktor „mediale Beziehungsdimension“ erklärt 31 Prozent der Gesamtvarianz. Das Item „Wenn es möglich wäre, würde ich [den Lieblings-Avatar] gerne einmal persönlich kennen lernen“ lässt sich im Rahmen dieses Faktors nur schlecht interpretieren. Eine inhaltliche Verbindung zur medialen Beziehungsdimension könnte man darin sehen, dass das Item eine Differenzierung zwischen „persönlicher Bekanntschaft“ und „medial vermittelter Bekanntschaft“ impliziert: Weil der Lieblings-Avatar nur aus den Medien bekannt ist, kann überhaupt erst der Wunsch entstehen, ihn persönlich kennen zu lernen.

Die Mittelwerte der beiden Beziehungsdimensionen weichen kaum vom geringen Durchschnittswert der Gesamtskala ab, wobei die mediale Beziehungsdimension $(M=2.07, S D=.99$, Cronbachs $\alpha=.89)$ etwas intensiver ausfällt als die quasi-reale $(M=1.50, S D=.79$, Cronbachs $\alpha=.86)$. Die höhere Ausprägung der medialen Dimension findet sich auch wieder, wenn man nur die Personen betrachtet, die sich für den gleichen Lieblings-Avatar entschieden. Bei Befragten, die Lara Croft als bevorzugten Avatar angegeben haben, ist die mediale Beziehungsdimension ausgeprägter $(M=2.10$, $S D=1.00)$ als die quasi-reale Dimension $(M=1.53, S D=.83)$. Der Unterschied zwischen medialer $(M=2.02, S D=1.00)$ und quasi-realer $(M=1.46, S D=.78)$ Beziehungsdimension findet sich ebenso bei dem männlichen Star-Avatar E-Cyas. Insofern macht sich die Abhängigkeit virtueller Akteure von ihrer medialen Vermittlung im empirischen Beziehungsmuster bemerkbar: Die parasozialen Bindungen zu Avataren sind eher durch das Bewusstsein der Medialität geprägt und besitzen kaum die Qualitäten einer „echten“ sozialen Beziehung.

Die zentrale Größe, die mediale Beziehungen zu Avataren fördert, ist deren wahrgenommene Attraktivität. Wer einen virtuellen Akteur als attraktiv beschreibt, neigt auch $\mathrm{zu}$ intensiveren medialen Beziehungen mit ihm oder ihr $(r=.49, p<.01)$, ist also motiviert, die Medienangebote, in denen der Avatar erscheint, zu nutzen, wobei dessen oder deren Aussehen allein noch keine „Freundschaft“ oder enge Bindung verursacht. Denn im Vergleich dazu spielt die Attraktivitätseinschätzung für die quasi-reale Beziehungsdimension eine geringere Rolle $(r=.28, p<.01)$.

Die Rolle des Geschlechts von Rezipienten und Persona. Die Intensität der zwei extrahierten Beziehungsdimensionen lässt sich anhand der Mittelwerte der jeweils zugeordneten Items bestimmen. Vergleicht man die Intensität der Beziehungsdimensionen von Frauen und Männern zu weiblichen bzw. männlichen Star-Avataren, so ergeben sich vier mögliche Verknüpfungen. Innerhalb der medialen Beziehungsdimension besitzen Männer die intensivsten Beziehungen zu weiblichen Star-Avataren, vor Beziehungen von Frauen zu männlichen Star-Avataren. Etwas schwächere Beziehungen liegen ferner von Frauen zu weiblichen Star-Avataren vor, und die schwächsten Beziehungen innerhalb der medialen Beziehungsdimension besitzen Männer zu männlichen Star-Avataren (vgl. Tabelle 2). Eine Varianzanalyse bestätigt den Interaktionseffekt $(F(1,333)=9.56, p<.01)$. Haupteffekte für das Geschlecht der Befragten $(F(1,333)=1.69$, 
$n s)$ und für das Geschlecht des Avatars $(F(1,333)=.26, n s)$ können hingegen nicht ausgemacht werden.

Tabelle 2: Intensität der medialen parasozialen Beziebung (Mittelwerte auf einer Skala von eins bis fünf) in Abhängigkeit vom Geschlecht der Befragten und des Lieblings-Avatars.

\begin{tabular}{lcc}
\hline Geschlecht der Befragten & $\begin{array}{c}\text { Mediale parasoziale } \\
\text { Beziehung zu männlichen } \\
\text { Avataren (M und SD; } \\
\text { Anzahl der Fälle) }\end{array}$ & $\begin{array}{c}\text { Mediale parasoziale } \\
\text { Beziehung zu weiblichen } \\
\text { Avataren (M und SD; } \\
\text { Anzahl der Fälle) }\end{array}$ \\
\hline Männlich & 1.75 & 2.30 \\
& $(.86)$ & $(.95)$ \\
& $\mathrm{n}=35$ & $\mathrm{n}=107$ \\
\hline Weiblich & 2.10 & 1.89 \\
& $(1.04)$ & $(1.00)$ \\
& $\mathrm{n}=107$ & $\mathrm{n}=93$ \\
\hline
\end{tabular}

Innerhalb der quasi-realen Beziehungsdimension findet sich dagegen weder ein Interaktionseffekt zwischen dem Geschlecht der Befragten und des Star-Avatars $(F(1,333)$ $=.61, n s)$, noch ergeben sich Haupteffekte für das Geschlecht der Befragten $(F(1,333)$ $=.03, n s)$ oder das Geschlecht des Avatars $(F(1,333)=.26, n s)$.

\section{Diskussion}

Die parasozialen Beziehungen der Befragten zu ihrem selbst ausgesuchten LieblingsAvatar sind nur schwach ausgeprägt. Es finden sich keine Hinweise darauf, dass virtuelle Akteure bereits das Potenzial besitzen, feste „Freunde“ von Internetnutzern zu werden. Die Rezipienten wahren ihnen gegenüber eine gewisse Distanz, wobei sie die Erscheinung der virtuellen Figuren sehr wohl als attraktiv empfinden können. Dennoch gibt es Anzeichen dafür, dass auch zu virtuellen Akteuren Ansätze parasozialer Beziehungen entstehen. Die Dimensionierung der parasozialen Beziehung in quasi-reale Beziehungsdimension einerseits und mediale Beziehungsdimension andererseits entsprechen recht gut der Einteilung, die Vorderer (1998) vornimmt. Insofern legt die gefundene Zwei-Faktoren-Lösung nahe, dass zu Avataren strukturell ähnliche Beziehungsmuster bestehen wie zu Fernsehfiguren. Dafür spricht auch, dass gegengeschlechtliche parasoziale Beziehungen etwas intensiver zu sein scheinen als gleichgeschlechtliche, was ebenfalls ein aus dem Fernsehbereich bekanntes Muster (Vorderer \& Knobloch, 1996) darstellt. Avatare sind also offensichtlich in der Lage, Medienpublika an sich zu binden wenn auch nicht sehr fest -, jedoch nicht, indem sie starke parasoziale Beziehungen knüpfen, sondern indem sie ästhetische Präferenzen bedienen, zum Beispiel das Modell eines schönen Menschen verkörpern. Diese Erkenntnisse können jedoch noch nicht als gesichert gelten, weil die Mittelwerte der verschiedenen Avatare auf der Skala zu parasozialen Beziehungen sehr gering ausfallen. Zukünftige Untersuchungen müssen hier weitere Klärung erbringen. 


\section{Studie 2}

\section{Fragestellung}

Studie 2 konzentrierte sich auf einen einzigen virtuellen Akteur, nämlich auf Lara Croft, die Protagonistin der mittlerweile fünfteiligen Computerspiele-Reihe Tomb Raider. Sie dürfte neben dem Videospiel-Helden Mario die bekannteste virtuelle Figur sein, denn zum einen gehören die Tomb Raider-Spiele zu den meist verkauften Titeln weltweit, und zum anderen ist Lara Croft auch in zahlreichen anderen Medienangeboten in Erscheinung getreten: Sie „spielt“ in einem Musikvideo („Männer sind Schweine“) mit, gibt „Interviews', füllt dank ihrer „körperlichen“ Erscheinung ganze Bilderstrecken in Zeitschriften und ziert zahlreiche Fanposter (vgl. auch Rettberg, 1999). Insofern kann sie als Mischform aus Stellvertreter- und Star-Avatar begriffen werden, denn während des Computerspielens agiert sie unter der vollständigen Kontrolle durch die Spieler, bei ihren sonstigen Medienauftritten wird sie dagegen als selbstständige Persona inszeniert. Es liegt daher nahe zu vermuten, dass Lara Croft den Mediennutzern und Computerspielern „Angebote“ für parasoziale Beziehungen unterbreitet. Sie könnte ebenso als beliebter und bewunderter "Star" fungieren wie zum Beispiel die Action-Helden aus Kino oder Fernsehen. In der Studie wurde deshalb ein Vergleich der parasozialen Beziehung zu Lara Croft mit der Bindung an eine beliebte Figur aus Film oder Fernsehen angestellt. Ziel der Untersuchung war es herauszufinden, inwiefern die parasozialen Beziehungen zu einer - besonders bekannten - virtuellen Figur hinsichtlich ihrer Intensität und Qualität den parasozialen Bindungen zu einer besonders populären Fernsehfigur ähnlich sind.

\section{Methode}

Im Rahmen einer größeren Studie über Computer- und Videospiele (vgl. auch Klimmt, 2001; Klimmt \& Vorderer, 2001) wurden die parasozialen Beziehungen zu Lara Croft und zu einer von den Teilnehmern genannten Lieblingsfigur aus Film oder Fernsehen erhoben. Dazu kam die in der Forschung über parasoziale Beziehungen bewährte Skala von Rubin, Perse und Powell (1985) in der modifizierten Form von Vorderer und Knobloch (1996) zum Einsatz. Durch die Modifikationen sollte die 20-Item-Skala speziell die Qualität der parasozialen Beziehungen besser erfassen. Die Teilnehmer füllten die Skala zunächst in Bezug auf Lara Croft aus, nannten anschließend ihre bevorzugte Figur aus Film oder Fernsehen und beantworteten dann die Skala erneut, dieses Mal in Bezug auf die genannte Film- bzw. Fernsehfigur. Für den Durchlauf, der Lara Croft betraf, wurden einige Items so umformuliert, dass sie besser zum Kontext der Computerspiele passten. So wurde beispielsweise das Item „Ich freue mich darauf, [Name der Lieblingsfigur] in der nächsten Folge wieder zu sehen " umformuliert in „Ich freue mich darauf, Lara Croft in der nächsten Tomb Raider-Folge wieder zu sehen“" (siehe Anhang). In beiden Varianten waren die Endpunkte der Likert-Skala mit „trifft überhaupt nicht zu“ bzw. „trifft voll und ganz zu“ bezeichnet. Zusätzlich wurde abgefragt, wie viele Tomb Raider-Titel die Teilnehmer besaßen und wie sie diese bewerteten. Der Fragebogen wurde an 5000 Personen aus der Kundendatenbank eines großen ComputerspieleUnternehmens verschickt. Er umfasste insgesamt elf Seiten. Unter den Teilnehmern wurden zahlreiche Preise verlost. Die Fragen konnten auf Wunsch auch online beantwortet werden; auf den wortgleichen Internet-Fragebogen wurde im Anschreiben hingewiesen. 


\section{Ergebnisse}

Insgesamt schickten 349 Personen den Fragebogen ausgefüllt zurück (Rücklaufquote: sieben Prozent). Die überwiegende Mehrheit (95 Prozent) der Teilnehmer war männlich; das Durchschnittsalter lag bei etwa 21 Jahren $(M=20.76$ Jahre; $S D=8.91$ Jahre). Rund 26 Prozent der Befragten nutzte das Internet zur Beantwortung. Lieblingsfiguren aus Film und Fernsehen nannten 325 Teilnehmer. Unter den 131 genannten Personae befanden sich höchst unterschiedliche Charaktere, zum Beispiel das Pokémon Pikachu und Marcel Reich-Ranicki. Mit Abstand am häufigsten genannt wurden Bart und Homer Simpson aus der Zeichentrickserie „Die Simpsons“. Es folgten Al Bundy sowie Agent Moulder aus der Mystery-Serie „Akte-X“. Die Befragten besaßen im Durchschnitt zwei $(M=2.06$; $S D=1.36)$ der zum Erhebungszeitpunkt erhältlichen vier Tomb Raider-Titel; nur knapp 15 Prozent der Teilnehmer besaßen kein eigenes Tomb RaiderSpiel. Insofern war der Avatar Lara Croft in der Stichprobe sehr gut bekannt.

Die Skalen zur parasozialen Interaktion erzielten zufriedenstellende Reliabilitätswerte. Sowohl für Lara Croft (Cronbachs $\alpha=.94)$ als auch im Durchlauf für die Lieblingsperson aus Film und Fernsehen (über alle genannten Figuren hinweg: Cronbachs $\alpha=.90$ ) ergeben sich akzeptable interne Konsistenzen. Die Auswertung beinhaltete vier Schritte. Zunächst wurden die Skalen zur parasozialen Beziehung zu Mittelwert-Indizes zusammengefasst und für Lara Croft und die genannte TV-Lieblingsfigur gegenübergestellt. Anschließend wurden die Lieblings-TV-Personen nach ihrem Geschlecht getrennt betrachtet und die parasozialen Bindungen zu ihnen erneut mit Lara Croft verglichen. Im dritten Schritt wurde eine Subgruppe von TV-Personae Lara Croft gegenübergestellt, und zwar die Gruppe der Zeichentrick-Charaktere. Sie sind hinsichtlich ihrer Darstellungsform ähnlich „abstrakt“ wie die Computerfigur Croft und wurden deswegen einem gesonderten Vergleich unterzogen. Im letzten Schritt wurden Zusammenhänge zwischen dem Ausmaß der parasozialen Beziehung zu Lara Croft und bestimmten in der bisherigen Forschung als zentral erachteten Merkmalen wie Dauer der „Bekanntschaft“ zur Persona betrachtet.

Vergleich der Indizes. Das Ausmaß der parasozialen Beziehung zu Lara Croft $(M=2.14 ; S D=.80)$ fällt geringer aus als zur Lieblingsfigur aus Film und Fernsehen $(M=2.60 ; S D=.80)$. Der Unterschied ist signifikant $(t(321)=-11.43 ; p<.01)$. Wie schon in Studie 1 erreicht der virtuelle Akteur also nur einen geringen Wert in der Skala zur parasozialen Beziehung und bleibt zusätzlich auch deutlich hinter der Lieblings-TV-Figur zurück.

Vergleich von weiblichen und männlichen TV-Personen mit Lara Croft. Angesichts des betont bzw. übertrieben weiblichen Aussehens von Lara Croft lag es nahe, den Vergleich der parasozialen Beziehungen zu verfeinern, indem das Geschlecht der TV-Person berücksichtigt wird (vgl. Tabelle 3). Da die überwiegende Mehrheit der Befragten männlich ist, konnte eine Geschlechterdifferenzierung auf Seiten der Teilnehmer nicht realisiert werden.

Die parasozialen Beziehungen zu weiblichen TV-Figuren erzielten den höchsten Wert im Indexvergleich. Vorderer und Knobloch (1996) fanden ebenfalls, dass männliche Befragte starke Bindungen zu weiblichen TV-Figuren wie Pamela Anderson berichten. Die Beziehung zu Lara Croft fällt im Vergleich zu den weiblichen TV-Figuren deutlich schwächer aus. Bei den Personen, die eine männliche TV-Figur bevorzugten, besteht dieser Abstand zwischen TV-Figur und Lara Croft ebenfalls; jedoch liegen die Werte im Vergleich zu der Subgruppe mit weiblichen Lieblingsfiguren etwa einen halben Skalenpunkt niedriger - sowohl für die TV-Figur als auch für Lara Croft. Insofern lässt sich 
Tabelle 3: Vergleich der parasozialen Beziehung zu Lara Croft und zur Lieblings-TVFigur, aufgeschlüsselt nach Geschlecht der genannten TV-Figur

\begin{tabular}{lcccc}
\hline & $\begin{array}{l}\text { Mittelwert PSB- } \\
\text { Index zu Lara } \\
\text { Croft (SD) }\end{array}$ & $\begin{array}{l}\text { Mittelwert PSB- } \\
\text { Index zur TV- } \\
\text { Figur (SD) }\end{array}$ & $\begin{array}{l}\text { t-Wert im Ver- } \\
\text { gleich zu Lara } \\
\text { Croft (df) }\end{array}$ & $\alpha$ \\
\hline $\begin{array}{l}\text { Personen, die eine } \\
\text { weibliche Lieblings- }\end{array}$ & 2.49 & 2.97 & -4.64 & $\mathrm{p}<.01$ \\
$\begin{array}{l}\text { TV-Figur nannten } \\
(\mathrm{n}=48)\end{array}$ & $(.96)$ & $(.78)$ & $(47)$ & \\
\hline $\begin{array}{l}\text { Personen, die eine } \\
\text { männliche Lieblings- }\end{array}$ & 2.09 & 2.54 & -10.31 & $\mathrm{p}<.01$ \\
$\begin{array}{l}\text { TV-Figur nannten } \\
(\mathrm{n}=274)\end{array}$ & $(.77)$ & $(.78)$ & $(273)$ & \\
\hline
\end{tabular}

die parasoziale Beziehung zu Lara Croft eher mit der zu einer weiblichen TV-Figur als mit der Bindung an eine männliche Figur vergleichen. Bei genauer Betrachtung erreicht Lara Croft nämlich bei den Items aus der Skala „... ist erotisch“ und „Ich finde ... attraktiv" die höchsten Werte und wird dort nur von jungen TV-Heldinnen wie Buffy, der Dämonenjägerin übertroffen. Das von Vorderer und Knobloch (1996) berichtete Beziehungsmuster zwischen männlichen Rezipienten und weiblicher Persona scheint also auch auf Lara Croft zuzutreffen.

Vergleich der parasozialen Beziehungen zu Lara Croft mit den Beziehungen zu Zeichentrick-Figuren. Lara Croft unterscheidet sich von TV-Personae durch ihre vergleichsweise unrealistische Erscheinung. Dies gilt jedoch nicht im Vergleich zu einer Subgruppe von TV-Personae, nämlich zu Zeichentrickfiguren. Sie sind ebenfalls weniger genaue Abbilder von „echten“ Menschen. Daher werden die parasozialen Beziehungen zu Zeichentrickfiguren (u. a. Homer und Bart Simpson, Kenny und Stan aus South Park) mit den Bindungen an Lara Croft verglichen. Insgesamt wählten 87 Befragte eine Zeichentrickfigur als Lieblings-Persona. In dieser Subgruppe unterscheiden sich die Index-Werte für Lara Croft $(M=2.07, S D=.77)$ und die Lieblings-TV-Figur $(M=2.18$, $S D=.66)$ nur geringfügig $(t(84)=-1.79, n s)$. Zum Vergleich: Der Index-Wert der parasozialen Beziehung zu den übrigen (fotorealistischen) Personae $(M=2.77, S D=.77)$ liegt deutlich höher als der Wert für die Zeichentrickfiguren $(t(317)=6.24, p<.01)$. Insofern lässt sich die parasoziale Bindung an Lara Croft eher mit der an Zeichentrickfiguren als mit der an TV-Personae aus Fleisch und Blut vergleichen.

Korrelate von parasozialen Beziehungen zu Lara Croft. In der bisherigen empirischen Forschung über parasoziale Beziehungen (im Überblick: Gleich, 1997; Vorderer, 1998) fanden sich immer wieder Zusammenhänge zwischen der parasozialen Beziehung zu TV-Personen und anderen Merkmalen, die auch Horton und Wohl (1956) schon postuliert hatten, zum Beispiel zwischen der parasozialen Beziehung und der Dauer der „Bekanntschaft" mit der Persona (vgl. Perse \& Rubin, 1989). Im letzten Auswertungsschritt wurden daher die Zusammenhänge zwischen dem Index-Wert der parasozialen Bindung an Lara Croft und der Anzahl der Tomb Raider-Spiele, die die Befragten besaßen, sowie der Bewertung dieser Spiele betrachtet. Je mehr Tomb Raider-Spiele eine Person besitzt, so ist zu vermuten, desto mehr Zeit hat sie auch mit Lara Croft verbracht, pflegt also eine länger andauernde „Bekanntschaft“ mit ihr. Darüber hinaus wurde untersucht, ob Personen, die intensivere parasoziale Bindungen an TV-Figuren berichten, auch zu einer stärkeren Bindung an Lara Croft neigen. Die Analyse dieser Zusammenhänge kann 
weitere Hinweise zu der Frage liefern, inwiefern sich das Verhältnis der Mediennutzer zum virtuellen Akteur Lara Croft mit dem zu einer TV-Persona vergleichen lässt. Ähnlich wie bei TV-Serienfiguren hängt auch bei Lara Croft das Ausmaß der parasozialen Beziehung mit der Länge des Kontakts zusammen. Die Anzahl der von den Befragten besessenen Tomb Raider-Spiele korreliert relativ hoch mit der parasozialen Bindung $(r=.42, p<.01)$. Auch die über 10er-Skalen erhobene Bewertung der Spiele weist einen deutlichen Zusammenhang mit der parasozialen Beziehung zu Lara Croft auf (für Tomb Raider 3: $r=.48, p<.01$ und für Tomb Raider 4: $r=.43, p<.01$ ). Schließlich zeigt sich ein deutlicher Zusammenhang zwischen der parasozialen Beziehung zu Lara Croft und der Beziehung mit der Lieblings-TV-Figur $(r=.60, p<.01)$. Wer sich also auf eine intensive Bindung an eine Fernsehfigur einlässt, fühlt sich auch eher dem virtuellen Akteur Croft verbunden.

\section{Diskussion}

Die virtuelle Akteurin Lara Croft ist offensichtlich nicht in der Lage, parasoziale Beziehungen anzubieten, deren Intensität mit den Bindungen zu Film- oder Fernsehfiguren vergleichbar ist. Gleichwohl lassen sich Anzeichen zumindest für schwache Bindungen finden. So wirkt sich die wahrgenommene Attraktivität, die bereits bei TV-Heldinnen für intensivere Beziehungen sorgt, auch auf die Relation zu Lara Croft aus. Die nicht fotorealistische, sondern gezeichnete Darstellungsform der Figur Croft macht sie hinsichtlich der Beziehungsintensität vergleichbar mit TV-Zeichentrickfiguren und unterscheidet sie deutlich von „echten“ TV-Personae. Schließlich finden sich bei den parasozialen Bindungen an Croft Korrelate, die als typisch für die Bindung an Personae gelten, nämlich die Dauer der Bekanntschaft und die positive Bewertung des Medienangebots, in dem die Persona auftritt. Insofern hat die Studie einige Hinweise darauf geliefert, dass Computerspieler parasoziale Beziehungen zu einer virtuellen Akteurin entwickeln, dass diese Bindungen jedoch deutlich schwächer ausgeprägt sind als bei Fernsehcharakteren und vor allem von der von männlichen Mediennutzern empfundenen Attraktivität der weiblichen Persona Croft getragen werden. Jedoch ist es durchaus denkbar, dass sich die gefundenen Intensitätsunterschiede zwischen Lara Croft und der Fernseh-Figur einebnen, wenn sich die Befragten nicht nur ihre LieblingsTV-Figur, sondern auch ihre Lieblings-Spiel-Figur aussuchen dürfen. In der vorliegenden Studie war Lara Croft als Spiel-Figur vorgegeben worden und muss deswegen nicht zwangsläufig bei den Befragten so populär gewesen sein wie die selbst gewählte Lieblings-TV-Figur. Andererseits handelte es sich bei der Stichprobe um überwiegend intensive Nutzer von Tomb Raider-Spielen, so dass insgesamt ein Vergleich zwischen Croft und Fernsehfigur auch nicht völlig unangemessen erscheint.

\section{Schlussbetrachtung und Ausblick}

Um die Frage zu klären, wie Mediennutzer mit virtuellen Akteuren umgehen, erweist sich der Zugang der parasozialen Beziehungen (Horton \& Wohl, 1956; Horton \& Strauss, 1957) theoretisch und empirisch als fruchtbar. Für diese Bewertung spricht, dass sich die Dimensionalisierung der in Studie 1 angewendeten Skala mit der Einteilung in anderen Untersuchungen zu parasozialen Beziehungen (Visscher \& Vorderer, 1998; Vorderer, 1998) deckt und dass sich in Studie 2 Zusammenhänge zwischen der parasozialen Beziehung zu Lara Croft, der Dauer der „Bekanntschaft“ und der Bewertung der Tomb Raider-Spiele finden, was ebenfalls mit den Ergebnissen aus dem Fernsehbe- 
reich korrespondiert (Perse \& Rubin, 1989). Es ergeben sich also in den Daten strukturelle Ähnlichkeiten zu den Erkenntnissen der Forschung über parasoziale Beziehungen zu Fernseh-Personae.

Die beiden vorgestellten Befragungsstudien über parasoziale Bindungen an Avatare zeigen, dass virtuelle Akteure, wie sie heute im Internet und in Computerspielen zum Einsatz kommen, in der Perspektive der Rezipienten eine andere Kategorie darstellen als Figuren aus Film und Fernsehen. Die parasozialen Beziehungen sind wenig intensiv und von der Medialität, der Abhängigkeit des virtuellen Akteurs von der Darstellung in einer künstlichen Medienumgebung, geprägt. So wahren die Mediennutzer eine gewisse Distanz zu den „neuen Prominenten“ wie E-Cyas oder Lara Croft. Von der geradezu überschwenglichen Bewunderung und Verehrung, wie sie typisch ist für Fans von TV-Stars (Leets, DeBecker \& Giles, 1995), sind die Beziehungen zu Avataren weit entfernt: Die Bezugspersonen (die Avatare) sind dem Namen und dem Aussehen nach den Mediennutzern bekannt, also kognitiv repräsentiert; sie besitzen jedoch nur eine geringe emotionale Relevanz.

Wenngleich Avatare für die Befragten beider Studien keine Bedeutung im Sinne von Freunden oder guten Bekannten haben, wissen die Mediennutzer doch die ästhetischen Qualitäten virtueller Akteure zu schätzen. Der Zusammenhang zwischen der Attraktivität des Avatars und der Stärke der „medialen“ parasozialen Beziehung wird in beiden Studien deutlich und ist auch aus der Forschung im Fernsehbereich bekannt (Gleich, 1997; Vorderer \& Knobloch, 1996). In diesem Zusammenhang ist auch das Ergebnis plausibel, dass gegengeschlechtliche parasoziale Beziehungen intensiver ausgeprägt sind als gleichgeschlechtliche.

Insgesamt scheinen also die heute verfügbaren Avatare in der Lage zu sein, den Rezipienten bzw. ihren „Zielgruppen“ zu gefallen, als bewunderte Stars oder gar Freunde eignen sie sich weniger. Es ist jedoch abzusehen, dass virtuelle Akteure in Zukunft vor allem an Interaktionskompetenz gewinnen werden (Bente \& Otto, 1996; Bente, Petersen, Krämer \& Buschmann, 1999). Während ihre äußere Erscheinung also bereits auf die Zustimmung der Rezipienten trifft und zur Publikumsbindung beiträgt, werden Avatare auch ihre „inneren“ Fähigkeiten verbessern. Auf diese Weise für den Dialog mit den Mediennutzern gerüstet, dürften virtuelle Akteure der kommenden Generationen auch als Bezugsperson für stärkere parasoziale Bindungen in Betracht kommen. Weil jedoch mit verbesserten Konversationsfähigkeiten das Wissen der Rezipienten um die Virtualität von Avataren nicht gelöscht werden kann, wird abzuwarten bleiben, bis zu welchem Maße sich die parasozialen Beziehungen zu ihnen tatsächlich denen zu menschlichen Prominenten angleichen werden.

Angesichts der zunehmenden Bedeutung von Virtual-Reality-Systemen in zahlreichen Anwendungsbereichen sollte die medienpsychologische Forschung mit der Entwicklung insbesondere virtueller Charaktere Schritt halten. Je mehr und je komplexere Avatare die digitalen Welten bewohnen, desto größere Erkenntnispotenziale bergen Untersuchungen darüber, wie Mensch und Avatar miteinander interagieren. Dies gilt nicht nur, aber auch für den Bereich der interaktiven Unterhaltung (Durkin \& Aisbett, 1999; Vorderer, 2000; Klimmt, 2001). Für entsprechende Studien müssen allerdings neue Erhebungsinstrumente entwickelt werden. An der in den vorgestellten Studien zugrunde gelegten Standard-Skala von Rubin, Perse und Powell (1985) wurde bereits im Kontext des ursprünglichen Einsatzgebiets, nämlich der TV-Figuren, Kritik geübt (Hippel, 1992; Gleich, 1997). Entsprechend stellt sich die Frage, inwiefern das Instrument für die Anwendung auf virtuelle Akteure geeignet ist. Bereits die hier beschriebenen ersten Annäherungen an parasoziale Beziehungen zu Avataren stießen an Grenzen der Skala, 
die zahlreiche Modifikationen unvermeidlich machten. In diesem Zusammenhang bietet es sich an, das Konzept der parasozialen Beziehungen sowohl in theoretischer Hinsicht als auch mit Blick auf seine empirische Messung stärker an die Erkenntnisse der Sozialpsychologie anzubinden (zu dieser Forderung vgl. Gleich, 1997). Denn wer die Interaktion zwischen Mensch und Avatar untersucht, betrachtet keine „Intimacy at a Distance“ (Horton \& Wohl, 1956/1986, S. 185) wie beim Fernsehen, sondern eine „direkte Interaktion mit wechselseitiger Kontingenz zwischen Mediennutzer und Bildschirmperson“ (Bente \& Otto, 1996, S. 225). Insofern kann das mittlerweile recht alte Konzept der parasozialen Beziehungen uns helfen, die Rezeption der neuen Medien zu verstehen. Eine Weiterentwicklung erscheint jedoch sowohl in theoretischer wie auch in methodischer Hinsicht dringend geboten.

\section{Literatur}

Bates, J. (1994). The Role of Emotions in Believable Agents (Online). Available: http:// riss.keris.or.kr:8080/pubs/citations/journals/cacm/1994-37-7/p122-bates (18.05.2000).

Bente, G. \& Otto, I. (1996). Virtuelle Realität und parasoziale Interaktion. Medienpsychologie, 8(3), $217-242$.

Bente, G., Petersen, A., Krämer, N. C. \& Buschmann, J.-U. (1999). Virtuelle Realität im Forschungseinsatz. Ein Wirkungsvergleich videovermittelter und computersimulierter nonverbaler Kommunikation. Medienpsychologie, 11(2), 95-120.

Blittkowsky, R. (1999). Echt virtuell. Nene Einsatzgebiete für Avatare. [online]. Verfügbar: http://rhein-zeitung.de/old/99/09/23/internet/news1.html (18.12.00).

Cassel, J. \& Vilhjálmsson, H. H. (1999). Fully Embodied Conversational Avatars: Making Communicative Behaviors Autonomous. Autonomous Agents and Multi-Agent-Systems, 2(1), 45 64.

Döring, N. (1999). Sozialpsychologie des Internet. Die Bedeutung des Internet für Kommunikationsprozesse, Identitäten, soziale Beziehungen und Gruppen. Göttingen: Hogrefe.

Durkin, K. \& Aisbett, K. (1999). Computer Games and Australians Today. Sydney: Office of Film and Literature Classification.

Eimeren, B. van \& Gerhard, H. (2000). ARD/ZDF-Online-Studie 2000: Gebrauchswert entscheidet über Internetnutzung. Media Perspektiven, o. J. (8), $338-349$.

Fabian, T. (1993). Fernsehen und Einsamkeit im Alter. Eine empirische Untersuchung zu parasozialer Interaktion. Münster: LIT Verlag.

Fritz, J. (2000). Schemata und Computerspiel. In Bundeszentrale für politische Bildung (Hrsg.), Computerspiele auf dem Prüfstand (Staffel 12, Nr. 94-98/2000) (S. 1-28). Bonn.

Gleich, U. (1996). Sind Fernsehpersonen die „Freunde“ des Zuschauers? Ein Vergleich zwischen parasozialen und realen sozialen Beziehungen. In P. Vorderer (Hrsg.): Fernsehen als „Beziehungskiste". Parasoziale Beziehungen und Interaktionen mit TV-Personen (S. 113-144). Opladen: Westdeutscher Verlag.

Gleich, U. (1997). Parasoziale Interaktionen und Beziehungen von Fernsehzuschauern mit Personen auf dem Bildschirm: ein theoretischer und empirischer Beitrag zum Konzept des aktiven Rezipienten. Landau: Verlag Empirische Pädagogik.

Hartmann, T. (2001). Cyber-Starlets. Eine empirische Untersuchung über parasoziale Beziehungen und Beziehungsqualitäten zu Star-Avataren wie E-Cyas oder Lara Croft. Unveröffentlichte Diplomarbeit an der Hochschule für Musik und Theater Hannover.

Hippel, K. (1992). Parasoziale Interaktion. Bericht und Bibliographie. montage/av, 1(1), 135 - 140.

Hippel, K. (1993). Parasoziale Interaktion als Spiel. Bemerkungen zu einer interaktionistischen Fernsehtheorie. montage/av, 2(2), $127-145$.

Höflich, J. R. (1998). Computerrahmen und die undifferenzierte Wirkungsfrage oder: Warum erst einmal geklärt werden muß, was die Menschen mit dem Computer machen. In P. Rössler, (Hrsg.), Online-Kommunikation. Wege zu Nutzung und Wirkung (S. 47-64). Opladen/Wiesbaden: Westdeutscher Verlag. 
Horton, D. \& Strauss, A. (1957). Interaction in audience-participation shows. The American Journal of Sociology, 62(6), $579-587$.

Horton, D. \& Wohl, R. R. (1956). Mass Communication and Para-social Interaction: Observation on Intimacy at a Distance. Psychiatry, 19, $215-229$.

Horton, D. \& Wohl, R. R. (1956/1986). Mass Communication and Para-social Interaction: Observation on Intimacy at a Distance. In G. Gumpert \& R. Cathcart (Hrsg.), Inter/Media. Interpersonal communication in a media world (S. 185 - 206). New York: Oxford University Press.

Jöckel, A. (1997). Virtuelle Sängerin wird zum Idol. [online]. Verfügbar: http://rheinzeitung.de/old/97/05/01/topnews/kyoko.html (18.12.00).

Keppler, A. (1996). Interaktion ohne reales Gegenüber. In P. Vorderer (Hrsg.), Parasoziale Beziebungen und Interaktionen mit TV-Personen (S. 11-24). Opladen: Westdeutscher Verlag.

Klimmt, C. (2001). Computer-Spiel: Interaktive Unterhaltungsangebote als Synthese aus Medium und Spielzeug. Zeitschrift für Medienpsychologie, 13(1), 22 - 32.

Klimmt, C. \& Vorderer, P. (2001, Januar). „Lara ist mein Medium“. Parasoziale Interaktionen mit Lara Croft im Vergleich zur Lieblingsfigur aus Film und Fernsehen. Vortrag auf der Jahrestagung der Fachgruppe Rezeptionsforschung der Deutschen Gesellschaft für Publizistik- und Kommunikationswissenschaft, 26. - 27.01.2001, Berlin.

Klussmann, N. (2000). Lexikon der Kommunikations- und Informationstechnik: Telekommunikation, Datenkommunikation, Multimedia, Internet (2., aktualisierte und überarbeitete Auflage). Heidelberg: Huethig.

Krotz, F. (1996). Parasoziale Interaktion und Identität im elektronisch mediatisierten Kommunikationsraum. In P. Vorderer (Hrsg.), Fernsehen als „Beziehungskiste“. Parasoziale Beziehungen und Interaktionen mit TV-Personen (S. 73 - 90). Opladen: Westdeutscher Verlag.

Leets, L., DeBecker, G. \& Giles, H. (1995). Fans: Exploring expressed motivations for contacting celebrities. Journal of Language and Social Psychology, 14(1-2), $102-123$.

Lombard, M. \& Ditton, T. (1997). At the Heart of it all: The Concept of Presence. Journal of Computer mediated Communication, 3(2) (Online). Available: http://209.130.1.169/jcmc/vol3/ issue2/lombard.html (18.05.2000).

Meyrowitz, J. (1978). Television and Interpersonal Behavior: Codes of Perception and Response. In G. Gumpert \& R. Cathcart (Hrsg.), Inter/Media. Interpersonal communication in a media world (S. 253 - 272). New York: Oxford University Press.

Moon, Y. \& Nass, C. (1996). How „Real“ are Computer Personalities? Psychological Responses to Personality Types in Human-Computer Interaction. Communication Research, 23(6), 651 674.

Perse, E. M. \& Rubin R. B. (1989). Attribution in social and parasocial relationships. Communication Research, 16, $59-77$.

Reeves, B. \& Nass, C. I. (1996). The Media Equation, How People Treat Computers, Television and New Media Like Real People and Places. Cambridge University Press.

Reilly, W. S. N. (1996). Believable Social and Emotional Agents. Technical Report CMU-CS-96138, School of Computer Science, Carnegie Mellon University, Pittsburgh, PA (Online). Available: http://www.cs.cmu.edu/afs/cs.cmu.edu/project/oz/web/papers.html (21.05.2000)

Rettberg, L. (1999). Zu schön um wahr zu sein. Die digitale Diva Lara Croft. montage/av, 8(2), 89 -110 .

Robinson, R. R. (1997). Creating Believable Agents in Computer Games (Online). Available: http://www.escape.ca/ rrrobins/Projects/ba-games.html (02.02.2000).

Rubin, R. B. \& McHugh, M. P. (1987). Development of Parasocial Interaction Relationships. Journal of Broadcasting E Electronic Media, 31(3), 279 - 292.

Rubin, A. M., Perse, E. M. \& Powell, R. A. (1985). Loneliness, parasocial interaction, and local television news viewing. Human Communication Research, 12(2), 155 - 180.

Schmidt, A. P. (1998). Avatare und ein Gespräch mit Bernhard Kolb, dem Geschäftsführer der IDMediengruppe. [online], Telepolis. Magazin der Netzkultur. Verfügbar: http://www.heise.de/ tp/deutsch/inhalt/co/2367/1.html (19.01.2001).

Snoddy, J. (2000). Reality Bytes. Attempt to make avatars more convincing. Guardian Unlimited (Online). Verfügbar: http://www.guardianunlimited.co.uk/efinance/article/0,2763,390424,00. html (09.02.2001). 
Stephenson, N. (1995). Snowcrash. München: Goldmann.

Trepte, S., Zapfe, S. \& Sudhoff, W. (im Druck). Orientierung und Problembewältigung durch TVTalkshows: Empirische Ergebnisse und Erklärungsansätze. Zeitschrift für Medienpsychologie.

Trzka, P. (1998). Die virtuelle Lotusblüte. [online]. Verfügbar: http://www. Bild.de/service.archiv/avatare/kyoko (03.02.2000).

Turing, A. M. (1950). Computing Machinery and Intelligence, Mind, 49, 433 - 460.

Ueberhorst, S. (1995). Avatar oder Agent. [online]. Verfügbar: http://www.computerwoche.de/archiv.cfm?path=http://www.computerwoche.de/archiv/1995/41/index.html (19.01.2001).

Vilhjálmsson, H. H. \& Cassel, J. (1998). Bodychat: Autonomous communicative behaviors in avatars. In Association for Computing Machinery (Hrsg.), Proceedings of the Second International Conference on Autonomous Agents (S. 269 - 277). New York.

Visscher, A. \& Vorderer, P. (1998). Freunde in guten und schlechten Zeiten. Parasoziale Beziehungen von Vielsehern zu Charakteren einer Daily Soap. In H. Willems \& M. Jurga (Eds.), Inszenierungsgesellschaft (S. 453 - 469). Opladen: Westdeutscher Verlag.

Vorderer, P. (Hrsg.) (1996a). Fernsehen als „Beziehungskiste“. Parasoziale Beziehungen und Interaktionen mit TV-Personen. Opladen: Westdeutscher Verlag.

Vorderer, P. (1996b). Picard, Brinkmann, Derrick und Co. als Freunde der Zuschauer. Eine explorative Studie über parasoziale Beziehungen zu Serienfiguren. In P. Vorderer (Hrsg.), Fernsehen als „Beziehungskiste“. Parasoziale Beziehungen und Interaktionen mit TV-Personen (S. 153 171). Opladen: Westdeutscher Verlag.

Vorderer, P. (1998). Unterhaltung durch Fernsehen: Welche Rolle spielen parasoziale Beziehungen zwischen Zuschauern und Fernsehakteuren? In G. Roters, W. Klingler \& O. Zöllner (Hrsg.), Fernsehforschung in Deutschland. Themen, Akteure, Methoden (S. 689 - 707). Baden-Baden: Nomos.

Vorderer, P. (2000). Interactive Entertainment and Beyond. In D. Zillmann \& P. Vorderer (Hrsg.), Media Entertainment: The Psychology of its Appeal (S. 21 - 36). Mahwah, NJ: Erlbaum.

Vorderer, P. \& Knobloch, S. (1996). Parasoziale Beziehungen zu Serienfiguren: Ergänzung oder Ersatz? Medienpsychologie, 8(3), S. $201-216$.

Wulff, H. J. (1996). Parasozialität und Fernsehkommunikation. Medienpsychologie, 8(3), S. 163 181.

\section{Anhang: Items zur parasozialen Beziehung (nach Vorderer \& Knobloch, 1996), in der für die Anwendung auf Lara Croft modifizierten Variante}

- Wenn ich Lara Croft auf dem Bildschirm sehe, kommt es mir vor, als wenn ich mit Freunden zusammen wäre, dann fühle ich mich wohl.

- Lara Croft ist erotisch.

- Ich freue mich darauf, Lara Croft in der nächsten „Tomb Raider“-Folge wiederzusehen.

- Wenn Lara Croft eine Figur in einem anderen Spiel wäre, würde ich es auf jeden Fall spielen.

- Ich habe den Eindruck, dass Lara Croft ähnliche Dinge wichtig sind wie mir.

- Wenn in Zeitungen oder Zeitschriften etwas über Lara Croft stünde, würde ich es auf jeden Fall lesen.

- Es kommt sogar vor, dass ich Lara Croft vermisse, wenn ich Tomb Raider längere Zeit nicht gespielt habe.

- Ich würde Lara Croft gerne einmal persönlich kennen lernen.

- Ich leide mit, wenn Lara Croft Probleme hat.

- Ich finde Lara Croft attraktiv.

- Manchmal überlege ich mir, wie ich mich verhalten würde, wenn ich in der gleichen Situation wäre wie Lara Croft. 
- In besonders spannenden Situationen kommt es schon mal vor, dass ich Lara Croft etwas zurufe, wie z. B. „Pass auf“.

- Wenn ich Probleme habe, überlege ich mir manchmal, wie Lara Croft sich selbst in meiner Situation verhalten würde.

- Manchmal vergleiche ich das Leben von Lara Croft mit dem Leben von Verwandten, Freunden und Bekannten.

- Ich bewundere Lara Croft.

- In vielen Punkten ähnelt das Leben von Lara Croft meinem Leben.

- Auf Lara Croft kann man sich verlassen.

- Ich wäre gerne wie Lara Croft.

- Manchmal vergleiche ich mein Leben mit dem Leben von Lara Croft.

- Ich empfinde Lara Croft als echte Person. 